\section{SLE: prognostischer Wert verschiedener \\ Remissions-Definitionen}

Saccon F et al. Remission in systemic lupus erythematosus: testing different definitions in a large multicentre cohort. Ann Rheum Dis 2020; 79: 943-950

Eine Remission bei Systemischem Lupus Erythematodes (SLE) wird von der Definitions of Remission In SLE (DORIS) task force anhand einer Kombination des clinical SLE Disease Activity Index (cSLE$D A I)=0$, des physician's global assessment $(P G A)<0,5$ und der täglichen Dosis Prednison (PDN) ( $\leq 5 \mathrm{mg} / \mathrm{d}$ ) klassifiziert. Eine italienische Studie hat die Performance dieser Items in Kombination und alleine in Bezug auf die Vorhersage des SDI Damage Index untersucht.

Während einer Follow-Up-Periode von $\geq 5$ Jahren testeten die Wissenschaftler 7 potenzielle Definitionen von Remissionen bei SLEPatienten, nämlich:

- PDN $\leq 5 \mathrm{mg} / \mathrm{d}$

- $P G A<0,5$

- CSLEDAI $=0$

- $P G A<0,5$ plus PDN $\leq 5 \mathrm{mg} / \mathrm{d}$

- cSLEDAI $=0$ plus PGA <0,5

- cSLEDAI $=0$ plus PDN $\leq 5 \mathrm{mg} / \mathrm{d}$

- $\quad$ CSLEDAI $=0$ plus PDN $\leq 5 \mathrm{mg} / \mathrm{d}$ plus $P G A<0,5$

Die Wissenschaftler bestimmten die negativen und positiven prädiktiven Werte der verschiedenen Remissions-Definitionen bezüglich der Vorhersage des Systemic Lupus International Collaborating Clinics/ACR Damage Index (SDI). Die beste Performance wurde als das niedrigste Akaike und Bayes'sche Informationskriterium (AIC und BIC) definiert.

In der Studie wurden Daten von 646 Patienten mit SLE ausgewertet, die mittlere Krankheitsdauer lag bei 9,2 $\pm 6,9$ Jahren. 90,6\% der Teilnehmer waren weiblich, das mittlere Alter lag bei 40,6 $\pm 12,1$ Jahren, die Krankheitsdauer lag bei 9,2 $\pm 6,9$ Jahren. Bei Studieneinschluss nahmen 84,4\% der Patienten Prednison, 71,2\% Anti-Malaria-Medikamente und 48,8\% Immunsuppressiva. Die Teilnehmer besuchten Ihre Ärzte mindestens 3-mal im Jahr und bei jedem Besuch wurde der SDI bestimmt. In der Auswertung wurde dann der Unterschied des SDI zu Beginn der Studie zum SDI nach 5 Jahren errechnet.

Zwischen 69,2\% und 88,7\% der Teilnehmer erreichten je nach Definition eine Remission von mindestens einem Jahr. Im fünfjährigen Beobachtungszeitraum erlitten 31,9\% neuen Schaden nach SDI.

Bei der multivariaten Analyse schützten $\geq 2$ Jahre Remission in Folge nach allen Definitionen vor Schädigung (OR, $95 \% \mathrm{KI}$ : PGA < 0,5 0,631, 0,444-0,896; cSLEDAI $=0$ 0,531, 0,371-0,759; PGA <0,5 plus PDN $\leq 5 \mathrm{mg} /$ Tag 0,554, 0,381-0,805; cSLEDAI = 0 plus PGA < 0,5 0,574, 0,400-0,826; cSLEDAI = 0 plus PDN $\leq 5 \mathrm{mg} /$ Tag 0,543, 0,376-0,785; cSLEDAI $=0$ plus PDN $\leq 5 \mathrm{mg} /$ Tag 0,543 , 0,376-0,785; cSLEDAI $=0$ plus PDN $\leq 5 \mathrm{mg} /$ Tag plus PGA $<0,5$ 0,532, 0,363-0,781, $\mathrm{p}<0,01$ für alle), außer PDN $\leq 5 \mathrm{mg} / \mathrm{d}$, wo 4 aufeinander folgende Jahre für eine Remission erforderlich waren (OR 0,534, $95 \% \mathrm{KI}$ 0,325-0,877, $p=0,013)$.

Die positiven und negativen prädiktiven Werte der unterschiedlichen Definitionen waren ähnlich; cSLEDAI $=0$ zeigte jedoch die beste Leistung (AIC 1082,90, BIC 1109,72, $\mathrm{p}<0,0001)$. Wenn PGA $<0,5$ und/oder PDN $\leq 5 \mathrm{mg} / \mathrm{d}$ zusätzlich zu cSLEDAI $=0$ in die Remissions-Definition eingeschlossen wurden, verringerte sich die Remissionsdauer ( $-1,8$ bzw. $-1,5$ Jahre/Patient), ohne dass sich die Leistung von cSLEDAI $=0$ bei der Vorhersage der Schadensentstehung erhöhte.

\section{FAZIT}

Die Autoren folgern, dass der leicht bestimmbare cSLEDAI sich als beste Remissionsdefinition bei SLE eignet und den Schadensverlaufs bei SLE am besten vorhersagen kann. Zusätzliche Kriterien verkürzen die Remissionsdauer, führen aber nicht zu einer genaueren Vorhersage künftiger Schadenereignisse.

Marisa Kurz M.Sc. B.A. München 